2. McNerney ME, Godley LA, Le Beau MM. Therapy-related myeloid neoplasms: when genetics and environment collide. Nat Rev Cancer. 2017;17:513-27.

3. Haddy N, Le Deley MC, Samand A, Diallo I, Guérin S, Guibout $\mathrm{C}$, et al. Role of radiotherapy and chemotherapy in the risk of secondary leukaemia after a solid tumour in childhood. Eur $\mathbf{J}$ Cancer. 2006;42:2757-64.

4. Hawkins M, Wilson L, Stovall M, Marsden H, Potok M, Kingston $\mathrm{J}$, et al. Epipodophyllotoxins, alkylating agents, and radiation and risk of secondary leukaemia after childhood cancer. BMJ. 1992;304:951-8.

5. Nottage K, Lanctot J, Li Z, Neglia JP, Bhatia S, Hammond S, et al. Long-term risk for subsequent leukemia after treatment for childhood cancer: a report from the Childhood Cancer Survivor Study. Blood. 2011;117:6315-8.

6. Tucker MA, Meadows AT, Boice Jr JD, Stovall M, Oberlin O, Stone BJ, et al. Leukemia after therapy with alkylating agents for childhood cancer. J Natl Cancer Inst. 1987;78:459-64.

7. Allodji RS, Schwartz B, Veres C, Haddy N, Rubino C, Le Deley M-C, et al. Risk of subsequent leukemia after a solid tumor in childhood: impact of bone marrow radiation therapy and chemotherapy. Int J Radiat Oncol Biol Phys. 2015; 93:658-67.

8. Green DM, Kun LE, Matthay KK, Meadows AT, Meyer WH, Meyers PA, et al. Relevance of historical therapeutic approaches to the contemporary treatment of pediatric solid tumors. Pedia Blood Cancer. 2013;60:1083-94.
9. Hudson MM, Neglia JP, Woods WG, Sandlund JT, Pui CH, Kun LE, et al. Lessons from the past: opportunities to improve childhood cancer survivor care through outcomes investigations of historical therapeutic approaches for pediatric hematological malignancies. Pedia Blood Cancer. 2012;58:334-43.

10. Bhatia S, Krailo MD, Chen Z, Burden L, Askin FB, Dickman PS, et al. Therapy-related myelodysplasia and acute myeloid leukemia after Ewing sarcoma and primitive neuroectodermal tumor of bone: a report from the Children's Oncology Group. Blood. 2007;109:46-51.

11. Ingram L, Mott M, Mann J, Raafat F, Darbyshire P, Jones PM. Second malignancies in children treated for non-Hodgkin's lymphoma and T-cell leukaemia with the UKCCSG regimens. Br J Cancer. 1987;55:463-6.

12. Pui C-H, Behm FG, Raimondi SC, Dodge RK, George SL, Rivera GK, et al. Secondary acute myeloid leukemia in children treated for acute lymphoid leukemia. N Engl J Med. 1989;321:136-42.

13. Winick NJ, McKenna RW, Shuster JJ, Schneider NR, Borowitz MJ, Bowman WP, et al. Secondary acute myeloid leukemia in children with acute lymphoblastic leukemia treated with etoposide. J Clin Oncol. 1993;11:209-17.

14. Inskip PD, Curtis RE. New malignancies following childhood cancer in the United States, 1973-2002. Int J Oncol. 2007;121:2233-40.

15. Rihani R, Bazzeh F, Faqih N, Sultan I. Secondary hematopoietic malignancies in survivors of childhood cancer: An analysis of 111 cases from the Surveillance, Epidemiology, and End Result-9 registry. Cancer. 2010;116:4385-94.

Leukemia (2019) 33:2951-2956

https://doi.org/10.1038/s41375-019-0526-5

Chronic lymphocytic leukemia

\title{
Diminished interaction between mutant NOTCH1 and the NuRD corepressor complex upregulates CCL17 in chronic lymphocytic leukemia
}

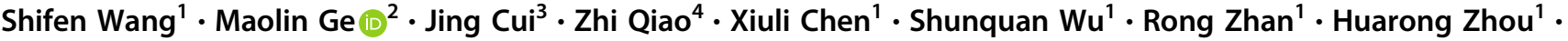 \\ Han Liu $\mathbb{( D}^{2} \cdot$ Zhenshu Xu ${ }^{1}$
}

Received: 25 January 2019 / Revised: 12 April 2019 / Accepted: 31 May 2019 / Published online: 24 July 2019

(c) The Author(s) 2019. This article is published with open access

These authors contributed equally: Shifen Wang, Maolin Ge, Jing Cui, Zhi Qiao

Supplementary information The online version of this article (https:// doi.org/10.1038/s41375-019-0526-5) contains supplementary material, which is available to authorized users.

Han Liu

liuhan68@sjtu.edu.cn

$\triangle$ Zhenshu Xu

zhenshuxu@yahoo.com

1 Fujian Institute of Hematology, Fujian Provincial Key Laboratory of Hematology, Fujian Medical University Union Hospital, 350001 Fuzhou, China

2 State Key Laboratory of Medical Genomics, Shanghai Institute of

\section{To the Editor:}

NOTCH1 mutation is one of the recurrent genetic lesions in chronic lymphocytic leukemia (CLL), the most common

Hematology, Rui Jin Hospital, School of Medicine and School of Life Sciences and Biotechnology, Shanghai Jiao Tong University, 200025 Shanghai, China

3 Department of Oncology, First Affiliated Hospital of Fujian Medical University, 350005 Fuzhou, China

4 State Key Laboratory of Microbial Metabolism, School of Life Sciences and Biotechnology, Shanghai Jiao Tong University, 200240 Shanghai, China 
leukemia in adult patients in Western countries [1-3]. NOTCH1 mutations associate with clinically aggressive forms of CLL and have emerged as an independent predictor of adverse prognosis [3]. Approximately $80 \%$ of NOTCH1 mutations in CLL consist of a 2-bp CT frameshift deletion (c.7541_7542delCT) that generates a truncation in the C-terminal PEST domain and prolongs the half-life of the active form of NOTCH1, i.e., the intracellular domain of NOTCH (ICN) [1, 2, 4-6]. There is evidence that the NOTCH1 mutation results in a truncated protein more stable than wild-type (WT) protein, thus sustaining active NOTCH1 signaling in CLL cells [4]. However, whether there is any functional difference between mutant and WT NOTCH1 remains largely unknown, and relatively little is known regarding why the most frequent NOTCH1 mutations in CLL occur in the PEST domain.

Recent studies have demonstrated that NOTCH1 mutations in CLL appear at the progenitor or pro-B cell stages [7], and might contribute to the expansion of CLL hematopoietic progenitors or pro-B cells [8]. CLL hematopoietic progenitors display higher levels of active ICN than their healthy counterparts $[9,10]$. Therefore, to investigate the functional difference between mutant and WT NOTCH1 in the B cell progenitors, we generated cell models mimicking CLL hematopoietic progenitors with high levels of ICN by ectopically expressing WT ICN (ICN) and mutant ICN (ICN-delCT) in the murine B cell progenitor Baf3 cell line [1], and determined whether they could differentially regulate the biological characteristics of these cells. Interestingly, even though ICN-delCT was expressed at a level comparable with ICN (Supplementary Fig. 1a), the Baf3 cells with ICN-delCT proliferated faster than those with WT ICN in a dose-dependent manner (Supplementary Fig. 1b), suggesting mutant NOTCH1 may cause more expansion in pro-B cells than WT NOTCH1.

Upon entering the nucleus, ICN forms a complex with the transcription factor CSL and the Mastermind-like family of co-activators to activate the transcription of a series of downstream genes such as HESI and DTX1 [11]. To determine whether WT and mutant ICN possess different transcriptional activities, we performed luciferase reporter assays in these cells using a 6xCSL-luciferase reporter. While both ICN and ICN-delCT significantly activated the reporter, ICN-delCT showed higher transcriptional activity than ICN (Fig. 1a). These results indicate that the enhanced pro-B cell expansion and elevated transcriptional activity are not caused by the prolonged half-life of the active form of NOTCH1, but rather reflect a functional difference between mutant and WT NOTCH1.

To identify the mechanisms underlying the functional difference between mutant and WT NOTCH1 in pro-B cells, we sought to determine whether ICN and ICN-delCT had different associated partners in the nucleus. ICN and its associated partners were purified from nuclear extracts derived from these Baf3 cells by immunoprecipitation using NOTCH1 antibody. Mass spectrometry analysis identified protein partners of ICN (Fig. 1b and Supplementary Table 1). Interestingly, consistent with our finding that ICNdelCT showed higher transcriptional activity, we found that MTA2 and MTA1 proteins, both of which are components of the nucleosome remodeling and deacetylase (NuRD) corepressor complex [12], co-purified with ICN but not with ICN-delCT (Supplementary Table 1). Factors involved in transcriptional repression, including components of the NuRD complex and the PRC1 complex, have been reported to be associated with ICN in T-cell acute lymphoblastic leukemia (T-ALL) cells [13]. However, because the primary function of ICN is to activate transcription, the biological significance of these interactions remains to be determined. To verify that the NuRD complex interacts with ICN but not ICN-delCT in B cells, we performed a coimmunoprecipitation experiment in primary cells from CLL patients and in these Baf3 cells and confirmed that ICN, but not ICN-delCT, associated with the NuRD complex components MTA2 and HDAC1 (Fig. 1c and Supplementary Fig. 1c). The diminished interaction between ICN-delCT and the NuRD corepressor complex might thus contribute to the elevated transcriptional activity of ICN-delCT in pro-B cells and over-activate a subset of genes critical for the survival of CLL cells, thus contributing to the pathogenesis of CLL.

Next, we asked if it was the truncation in the PEST domain that caused the diminished interaction between ICN-delCT and the NuRD complex. To this end, we examined the direct interactions between MTA2 and various PEST truncations using pull-down assays. The results showed that the truncated PEST domain from mutant NOTCH1 was no longer able to bind with MTA2, indicating the C-terminal portion of the PEST domain which is lost in mutant NOTCH1 was responsible for direct interaction with MTA2 (Fig. 1d). Previously, besides providing a degradation signal, other functions of the PEST domain in NOTCH1 had not yet been identified. However, our results suggest the PEST domain not only provides a degradation signal, but also participates actively in NOTCH1 function by recruiting critical protein partners such as the NuRD complex.

Because the NuRD corepressor complex interacts specifically with ICN but not with ICN-delCT in B cells, we hypothesized that at least a subset of dormant NOTCH1 target genes were not activated until NOTCH1 was mutated. To identify the NOTCH1 target genes that are preferentially activated by mutant NOTCH1, we performed gene expression profiling of these Baf3 cells. Gene ontology analysis showed that immune system processes were upregulated in Baf3 cells expressing ICN-delCT (Supplementary Fig. 2a). 
a

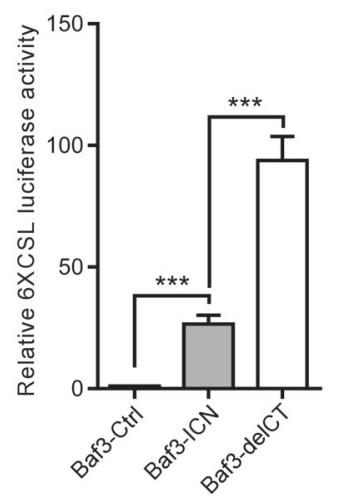

C
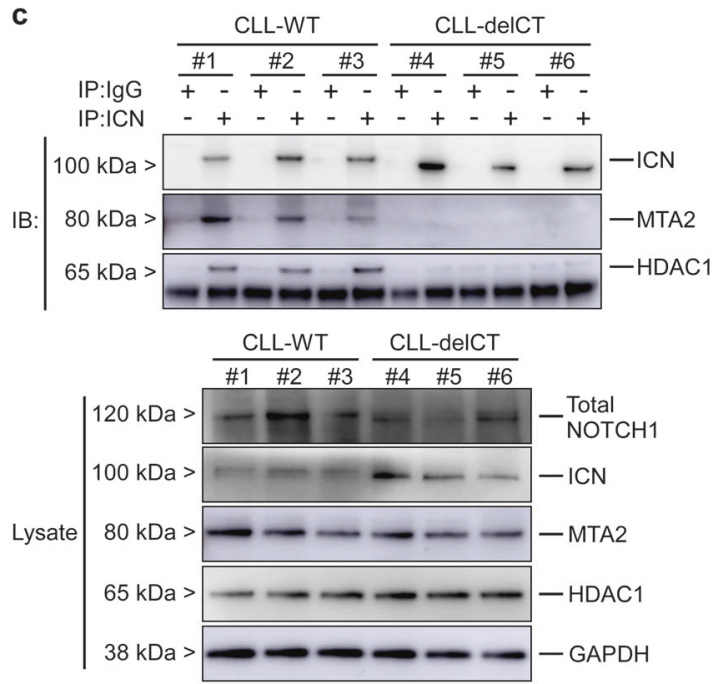

Fig. 1 ICN but not ICN-delCT interacts with NuRD corepressor complex. a The dual-luciferase reporter assay with the 6xCSL-luc reporter in Baf3 cells transfected with ICN/ICN-delCT plasmids or the empty vector as a control. Efficiency of transfection was normalized to Renilla luciferase. b The liquid chromatography-tandem mass spectrometry (LC-MS/MS) assay of the protein solution immunoprecipitated using anti-NOTCH1 antibody from Baf3 cells transfected with plasmids expressing GFP-tagged ICN/ICN-delCT as indicated. The part base peak spectra of the binding targets of MTA2 are shown. c The co-IP assay of the indicated primary cells of the CLL patients. Cellular lysates were immunoprecipitated with anti-ICN (cleaved

Consistently, the KEGG pathway enrichment analysis also identified that genes referring to cytokine-cytokine receptor interaction and the chemokine signaling pathway were significantly enriched in the ICN-delCT group (Supplementary Fig. 2b). In particular, genes encoding Ccl17 family chemokines, including the Ccll7 and Ccl22 genes, were significantly upregulated in the ICN-delCT group (Fig. 2a, b). These results indicated that the $\mathrm{Ccl} 17$ and $\mathrm{Ccl} 22$ genes as NOTCH1 target genes preferentially activated by mutant NOTCH1. In line with this, the mRNA level of CCL17 and CCL22 was significantly elevated in primary CLL cells with NOTCH1-delCT (Fig. 2c). We further reanalyzed the published RNA-seq data in CLL patients (GSE92626) [9], and confirmed that the transcriptional level

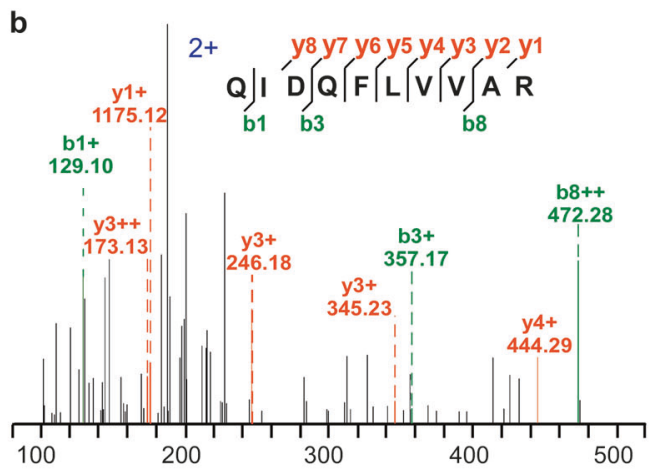

d
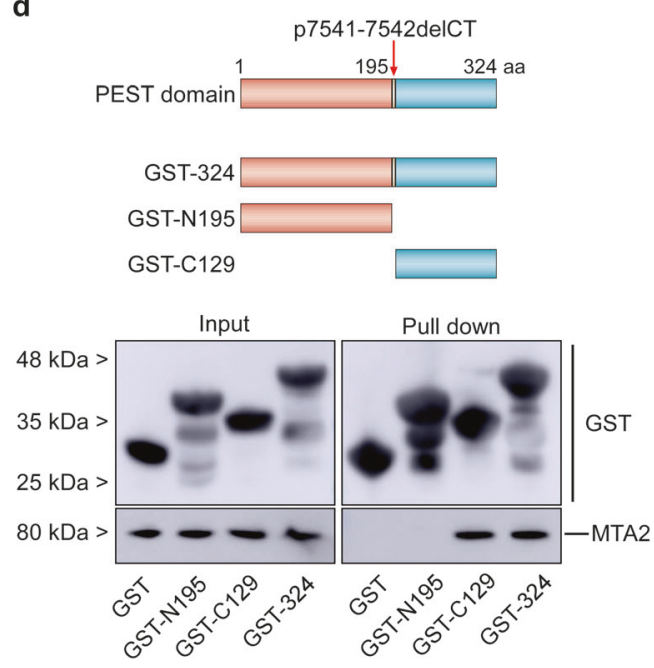

NOTCH1, Val1744) antibody (CST \#4147), then immunoblotted with anti-NOTCH1 (CST \#3608), anti-ICN (CST \#4147), anti-MTA2, or anti-HDAC1 antibodies, respectively. CLL-WT, CLL with WT NOTCH1; CLL-delCT, CLL with NOTCH1-delCT mutation. d The GST pull-down assay to measure the interaction of MTA2 with GST tagged PEST domain (GST-324), and PEST truncated fragment (GST-N195 and GST-C129). GST-fusion proteins were purified from E. coli, evaluated by immunoblotting, and incubated with eukaryotic expressed MTA2. Glutathione beads bound proteins were evaluated by western blotting with anti-MTA2 antibody. $* * * P<0.001$; two-tailed $t$-test. Data represent means of triplicate reactions \pm sem

of the CCL17 gene was elevated in human CLL cells with NOTCH1-delCT (Supplementary Fig. 2c, d).

To confirmthe pivotal role of the NuRD corepressor complex in suppressing the expression of CCL17 family genes, we knocked down the MTA2 gene in the primary CLL cells and observed that MTA2 depletion significantly induced the expression of the CCL17 and CCL22 genes in NOTCH1-WT but not NOTCH1-delCT CLL cells (Fig. 2d, e). In contrast, the mRNA level of other NOTCH1 target genes, such as HES1, HES4, and HEYl, was not significantly induced by MTA2 depletion (Supplementary Fig. 3a), indicating that only a subset of NOTCH1 target genes in CLL cells were repressed by the NuRD corepressor complex. To determine whether CCL17 gene transactivation 

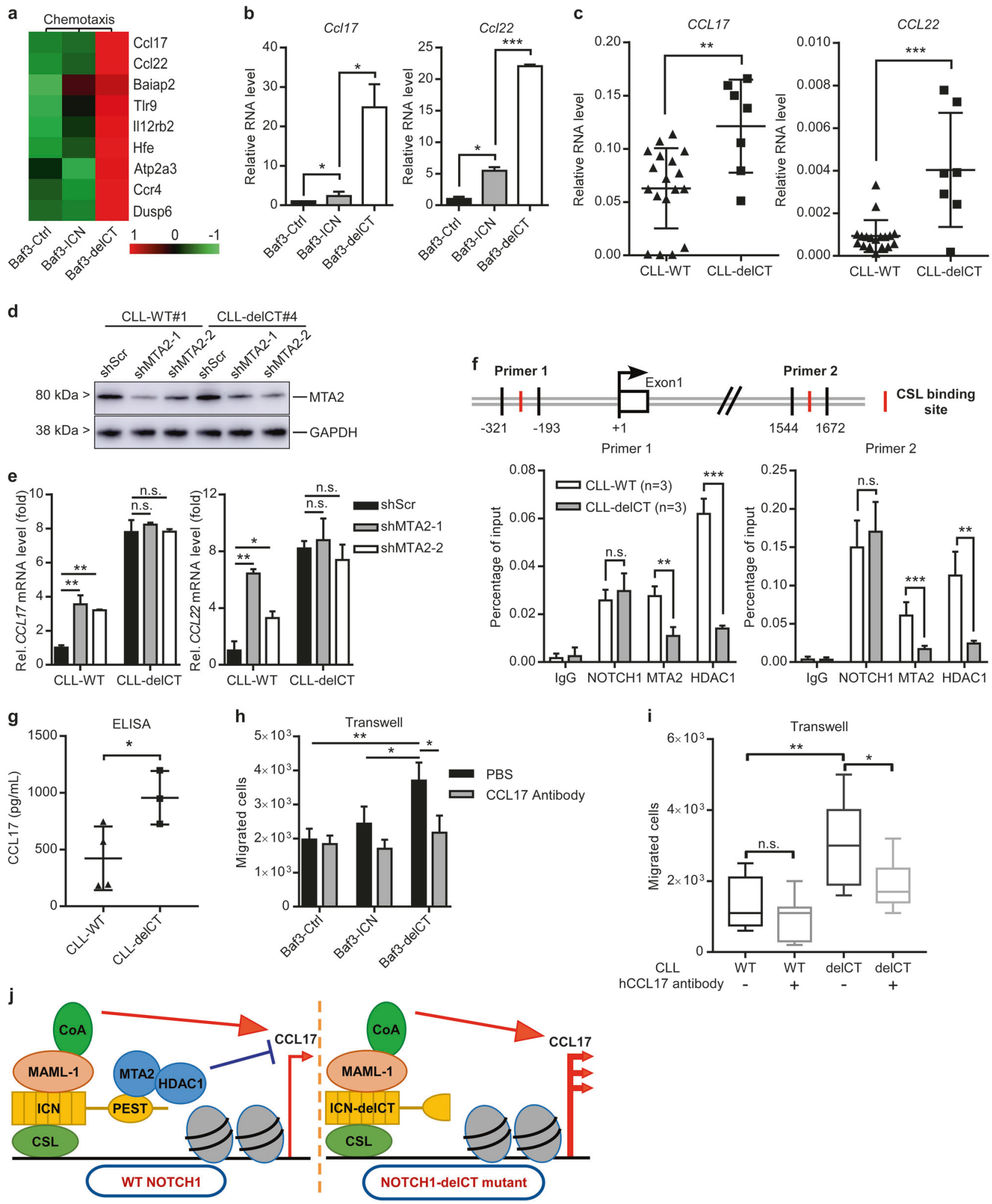

in primary CLL cells was associated with a diminished interaction between ICN-delCT and the NuRD complex, we examined the recruitment of MTA2 and HDAC1 onto the CSL-binding sites of the CCL17 gene using chromatin immunoprecipitation (ChIP) assays. Both WT ICN and ICN-
delCT showed a significant enrichment on the CSL-binding sites of the CCL17 gene; however, the enrichment of MTA2 and HDAC1 on these sites was significantly decreased in NOTCH1-delCT CLL cells (Fig. 2f). The diminished recruitment of the NuRD complex to the Ccll7 gene was 
Fig. 2 The loss of NuRD interaction with mutant NOTCH1 overactivates a subset of genes critical for the survival of CLL cells. a Heatmap showing the nine upregulated genes related to the cytokine signaling pathway in ICN-delCT variants. Color indicates normalized expression level (log); Red corresponds to higher expression, and green corresponds to lower expression. b $\mathrm{Ccl} 17$ and Ccl22 mRNA levels in Baf3 cells transfected with the indicated plasmids. Values were normalized against Gapdh. c The transcriptional level of CCL17 and $C C L 22$ genes in human CLL patients with WT NOTCH1 (CLLWT, $n=18$ ) or NOTCH1-delCT mutation (CLL-delCT, $n=7$ ). d Primary CLL cells were infected with the indicated lentiviral vectors. Immunoblots of the indicated proteins were detected. e CCL17 and CCL22 mRNA levels in primary CLL cells described in d. Values were normalized against GAPDH. f Schematic of the CCL17 promoter indicating the CSL-binding sites (upper panel). ChIP assays detected NOTCH1-ICN, MTA2, and HDAC1 on CCL17 gene promoter performed in primary cells of the indicated CLL patients (lower panel). ChIP results are presented as the mean of experiments performed in three independent samples. $\mathbf{g}$ The chemokine CCL17 concentration in the serum of human CLL patients with WT NOTCH1 or NOTCH1delCT was detected by ELISA. $\mathbf{h}$ The chemotaxis assay for cellular supernatants derived from Baf3 cells expressing ICN or ICN-delCT. Transwell chambers were used to detect T-cell migration ability and the number of migrated cells was counted manually. $\mathbf{i}$ The chemotaxis assay for the serum of human CLL patients with WT $(n=3)$ or NOTCH1-delCT $(n=3)$. j A schematic model illustrating the induction of CCL17 by NOTCH1-delCT mutation in CLL. CoA, coactivator. $* P<0.05 ; * * P<0.01 ; * * * P<0.001 ;$ n.s., not significant; two-tailed $t$-test. Data represent means of triplicate reactions \pm sem

also validated in ICN-delCT-expressing Baf3 cells (Supplementary Fig. 3b). Taken together, these results suggest that the diminished interaction between ICN-delCT and the NuRD complex is responsible for the transactivation of the CCL17 gene in the NOTCH1-delCT CLL cells.

Chemokine CCL17 plays a critical role in the survival of CLL cells by modulating the tumor microenvironment [14]. Malignant CLL cells have the capacity to attract $\mathrm{CD} 4^{+}$ T-cells expressing CCR4, the receptor for CCL17 and CCL22, which provide survival and growth signals to CLL cells [15]. In line with the elevated RNA level, ELISA assays also confirmed that chemokine CCL17 was significantly elevated in the serum of CLL patients with NOTCH1-delCT (Fig. 2g). Consistently, the chemokine Ccl17 was also significantly induced in the supernatant of ICN-delCTexpressing Baf3 cells (Supplementary Fig. 3c). Transwell assays further showed that the number of migrating $\mathrm{CD} 4^{+} \mathrm{T}-$ cells was notably increased by the media of ICN-delCTexpressing Baf3 cells and the serum of NOTCH1-delCT CLL patients (Fig. 2h, i), which could be effectively blocked by CCL17 antibody. Hence, the NOTCH1 mutation in CLL is related to the induction of CCL17 and subsequently induced the migration of $\mathrm{CD} 4^{+} \mathrm{T}$-cells, which changes the microenvironment to favor tumor cell survival.

NOTCH1 genetic alterations have been described in different human malignancies, including T-ALL and CLL. Nevertheless, the reason why NOTCH1 mutations affect two different domains in CLL and T-ALL remains unclear. Our study proposes that loss of NuRD complex interaction is a novel mechanism underlying the activation of NOTCH1 prevalent in CLL (Fig. 2j). Our study revealed that in CLL, the prevailing NOTCH1 mutants lacking an intact C-terminal PEST domain not only became more stable, but also gained additional function by becoming more potent transcriptional activators. Our study thus explains why NOTCH1 mutations in CLL preferentially occur in the PEST domain and provides new insights that will enable more precise therapeutic strategies specific for NOTCH1mutated CLL.

\section{Data availability}

RNA-seq raw data are available in the Gene Expression Omnibus database under accession number GSE115728.

Acknowledgements This work was supported by the National Key Research and Development Program of China (2018YFA0107802), the Joint Funds for the Innovation of Science and Technology of Fujian Province of China (no. 2017Y9005), the National Natural Science Foundation of China (81570119 and 81370651), the Program of Shanghai Academic Research Leader (19XD1402500), the Shanghai Municipal Health Commission (2019CXJQ01), the Shanghai Municipal Education Commission Gaofeng Clinical Medicine Grant (20161304), the Collaborative Innovation Center of Hematology, and the Samuel Waxman Cancer Research Foundation.

Author contribution S.W., M.G., J.C., and Z.Q. performed the experiments and contributed equally to this study; X.C., S.-Q.W., R.Z., and H.Z. assisted with some experiments; S.W., M.G., Z.X., and H.L. wrote the paper; and Z.X. and H.L. supervised the work.

\section{Compliance with ethical standards}

Conflict of interest The authors declare that they have no conflict of interest.

Publisher's note: Springer Nature remains neutral with regard to jurisdictional claims in published maps and institutional affiliations.

Open Access This article is licensed under a Creative Commons Attribution 4.0 International License, which permits use, sharing, adaptation, distribution and reproduction in any medium or format, as long as you give appropriate credit to the original author(s) and the source, provide a link to the Creative Commons license, and indicate if changes were made. The images or other third party material in this article are included in the article's Creative Commons license, unless indicated otherwise in a credit line to the material. If material is not included in the article's Creative Commons license and your intended use is not permitted by statutory regulation or exceeds the permitted use, you will need to obtain permission directly from the copyright holder. To view a copy of this license, visit http://creativecommons. org/licenses/by/4.0/.

\section{References}

1. Fabbri G, Rasi S, Rossi D, Trifonov V, Khiabanian H, Ma J, et al. Analysis of the chronic lymphocytic leukemia coding genome: role of NOTCH1 mutational activation. $J$ Exp Med. 2011;208:1389-401. 
2. Puente XS, Pinyol M, Quesada V, Conde L, Ordonez GR, Villamor $\mathrm{N}$, et al. Whole-genome sequencing identifies recurrent mutations in chronic lymphocytic leukaemia. Nature. 2011;475:101-5.

3. Rosati E, Baldoni S, De Falco F, Del Papa B, Dorillo E, Rompietti $\mathrm{C}$, et al. NOTCH1 aberrations in chronic lymphocytic leukemia. Front Oncol. 2018;8:229.

4. Arruga F, Gizdic B, Bologna C, Cignetto S, Buonincontri R, Serra $\mathrm{S}$, et al. Mutations in NOTCH1 PEST domain orchestrate CCL19driven homing of chronic lymphocytic leukemia cells by modulating the tumor suppressor gene DUSP22. Leukemia. 2017;31:1882-93.

5. De Falco F, Sabatini R, Falzetti F, Di Ianni M, Sportoletti P, Baldoni S, et al. Constitutive phosphorylation of the active Notch1 intracellular domain in chronic lymphocytic leukemia cells with NOTCH1 mutation. Leukemia. 2015;29:994-8.

6. Pozzo F, Bittolo T, Arruga F, Bulian P, Macor P, Tissino E, et al. NOTCH1 mutations associate with low CD20 level in chronic lymphocytic leukemia: evidence for a NOTCH1 mutation-driven epigenetic dysregulation. Leukemia. 2016;30:182-9.

7. Quijada-Alamo M, Hernandez-Sanchez M, Robledo C, Hernandez-Sanchez JM, Benito R, Montano A, et al. Nextgeneration sequencing and FISH studies reveal the appearance of gene mutations and chromosomal abnormalities in hematopoietic progenitors in chronic lymphocytic leukemia. J Hematol Oncol. 2017;10:83.
8. Kikushige $\mathrm{Y}$, Ishikawa $\mathrm{F}$, Miyamoto $\mathrm{T}$, Shima $\mathrm{T}$, Urata $\mathrm{S}$, Yoshimoto G, et al. Self-renewing hematopoietic stem cell is the primary target in pathogenesis of human chronic lymphocytic leukemia. Cancer Cell. 2011;20:246-59.

9. Fabbri G, Holmes AB, Viganotti M, Scuoppo C, Belver L, Herranz $\mathrm{D}$, et al. Common nonmutational NOTCH1 activation in chronic lymphocytic leukemia. Proc Natl Acad Sci USA. 2017;114:E2911-E2919.

10. Di Ianni M, Baldoni S, Del Papa B, Aureli P, Dorillo E, De Falco $\mathrm{F}$, et al. NOTCH1 is aberrantly activated in chronic lymphocytic leukemia hematopoietic stem cells. Front Oncol. 2018;8:105.

11. Bray SJ. Notch signalling in context. Nat Rev Mol Cell Biol. 2016;17:722-35.

12. Lai AY, Wade PA. Cancer biology and NuRD: a multifaceted chromatin remodelling complex. Nat Rev Cancer. 2011;11:588-96.

13. Yatim A, Benne C, Sobhian B, Laurent-Chabalier S, Deas O, Judde JG, et al. NOTCH1 nuclear interactome reveals key regulators of its transcriptional activity and oncogenic function. Mol Cell. 2012;48:445-58.

14. Yan XJ, Dozmorov I, Li W, Yancopoulos S, Sison C, Centola M, et al. Identification of outcome-correlated cytokine clusters in chronic lymphocytic leukemia. Blood. 2011;118:5201-10.

15. Ghia P, Strola G, Granziero L, Geuna M, Guida G, Sallusto F, et al. Chronic lymphocytic leukemia B cells are endowed with the capacity to attract $\mathrm{CD} 4^{+}, \mathrm{CD} 40 \mathrm{~L}^{+} \mathrm{T}$ cells by producing CCL22. Eur J Immunol. 2002;32:1403-13.

Leukemia (2019) 33:2956-2962

https://doi.org/10.1038/s41375-019-0523-8

Acute myeloid leukemia

\title{
TP53 abnormalities and chromosomal aneuploidy in acute panmyelosis with myelofibrosis
}

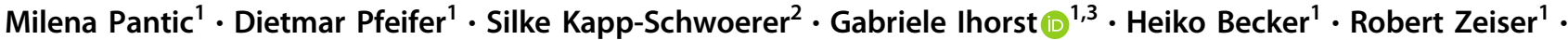 \\ Justus Duyster ${ }^{1} \cdot$ Annette Schmitt-Graeff $^{4}$
}

Received: 8 January 2019 / Revised: 16 April 2019 / Accepted: 9 May 2019 / Published online: 26 July 2019

(c) The Author(s) 2019. This article is published with open access

These authors contributed equally: Milena Pantic, Dietmar Pfeifer

Supplementary information The online version of this article (https:// doi.org/10.1038/s41375-019-0523-8) contains supplementary material, which is available to authorized users.

Annette Schmitt-Graeff

annette.schmitt-graeff@uniklinik-freiburg.de

1 Department of Hematology, Oncology and Stem Cell Transplantation, Medical Center - University of Freiburg, Faculty of Medicine, University of Freiburg, Germany, Freiburg, Germany

2 Department of Internal Medicine III, University Hospital of Ulm, Ulm, Germany

3 Clinical Trials Unit, Medical Center - University of Freiburg, Faculty of Medicine, University of Freiburg, Freiburg, Germany

4 University of Freiburg, Freiburg, Germany

\section{To the Editor:}

The World Health Organization (WHO) classification of acute myeloid leukemia (AML) has incorporated molecular genetic and cytogenetic aberrations in the definition of most entities [1]. The diagnosis of acute panmyelosis with myelofibrosis (APMF) is still not based on genomic changes but on clinicopathologic features and the exclusion of other myeloid malignancies, in particular AML with myelodysplasia-related changes (AML-MRC) [2]. APMF is a rapidly progressive hyperfibrotic subtype of "AML not otherwise specified" (AML NOS) and accounts for $<1 \%$ of AML cases [3]. This aggressive disease is characterized by rapid onset of cytopenia and constitutional symptoms in the absence of splenomegaly, previous history of myeloproliferative neoplasm (MPN) or myelodysplastic 